\title{
GROUND MOTION MEASUREMENTS FOR FERMILAB FUTURE COLLIDER PROJECTS
}

\author{
B. Baklakov, T. Bolshakov, A. Chupyra, A. Erokhin, P. Lebedev, V. Parkhomchuk, Sh. Singatulin, \\ BINP, Novosibirsk, Russia; and J. Lach, V. Shiltsev, FNAL, Batavia, IL, USA
}

\section{Abstract}

This article presents results of wide-band seismic measurements at the Fermilab site, namely, in the tunnel of the Tevatron and on the surface nearby, and in two deep tunnels in the Illinois dolomite, thought to be a possible geological environment of the Fermilab future accelerators.

\section{MEASUREMENTS}

There are several future collider projects under consideration at Fermilab, including muon collider, linear collider and Very Large Hadron Collider(VLHC). Ground motion is of concern for all of the projects, although due to different effects [1]. That was major reason for seismic studies at the Fermilab site and in the Illinois dolomite tunnels which we carried out in 1997. Our seismic instrumentation included eight modified geophone of SM3-KV type (frequency range from 0.07 to $120 \mathrm{~Hz}$ ) two tri-axial STS2 seismometers $(0.005-15 \mathrm{~Hz})$, and two Wiloxon-731A piezoaccelerometers $(10-400 \mathrm{~Hz})$.

Measurements at Fermilab Vibration measurements in the Tevatron tunnel have been done at Sector F11 (not far from the Tevatron RF station and the E4R building) and Sector F21 some $300 \mathrm{~m}$ apart. The computer was located on the surface in the F0 building. Seven SM3-KV probes (four vertical and three horizontal) and two vertical piezoaccelerometers were used. Two seismic stations were placed at a distance $296 \mathrm{~m}$ apart. Station 1 digitized the signals from one vertical and one horizontal SM3-KV probes on the floor of the tunnel at F21, and from vertically oriented piezoaccelerometer and vertical and horizontal SM3-KV geophones on the Tevatron quadrupole magnet. Station 2 digitized the signals from four SM3-KV geophones (vertical and horizontal on the quadrupole magnet at F11 and vertical and horizontal on the tunnel floor nearby), one piezoaccelerometer placed on the same magnet, and additionally from a beam position monitor (BPM) and a beam loss monitor (BLM). Recording vibration signals in the Tevatron tunnel over several days, we observed little day-night variation of the maximum tunnel floor motion amplitude. Cultural noise dominates in vibrations of the magnet and the tunnel floor. At frequencies above 1 $\mathrm{Hz}$ it is due to the technical equipment (water and helium pipes, power cables, magnets themselves, etc.) At frequencies around $1 \mathrm{~Hz}$ and lower the main contribution is possibly due to strong mechanical distortions of the magnets during the Main Ring acceleration cycle (about 3 s) and the Tevatron acceleration cycle (about $60 \mathrm{~s}$ in fixed target operation).
The closed orbit distortions are caused by the displacements of all magnetic elements along the circumference of Tevatron. We detected strong coherence between the magnet and beam vibrations. It means that there is a common source of vibration along the whole accelerator ring. For example, several remarkable peaks in the orbit-magnet coherence occur at $4.6 \mathrm{~Hz}, 9.2 \mathrm{~Hz}, 13.8 \mathrm{~Hz}$, etc., at the Fermilab site specific frequencies caused by Central Helium Liquefier plant operation.

Measurements in deep tunnels Specific locations for possible Fermilab Future Colliders have not yet been chosen. There is also no definite requirement to be located within the FNAL site. For the purposes of radiation safety and tunnel stability, deep tunnels in the Illinois dolomite layer are alternative. This several hundreds feet thick layer is considered as moderately hard and stable. We studied seismic vibrations at two points of the Illinois dolomite layer. The first is a $250 \mathrm{ft}$ deep mine (Conco Mine - Western Stone Co., North Aurora, IL; about 500ft above sea level) located about 5 miles North-West of Fermilab. The second location is a $300 \mathrm{ft}$ deep tunnel of the Metropolitan Water Reclamation District of Greater Chicago (MWRDGC) about 30 miles East of the Fermilab in the Chicago suburb of Hodgkins, IL. It is near $(<0.5$ mile $)$ from an active interstate highway I-55, and very close to a stone quarry. The tunnel was constructed as a part of the Tunnel and Reservoir Project (TARP) of the MWRDGC.

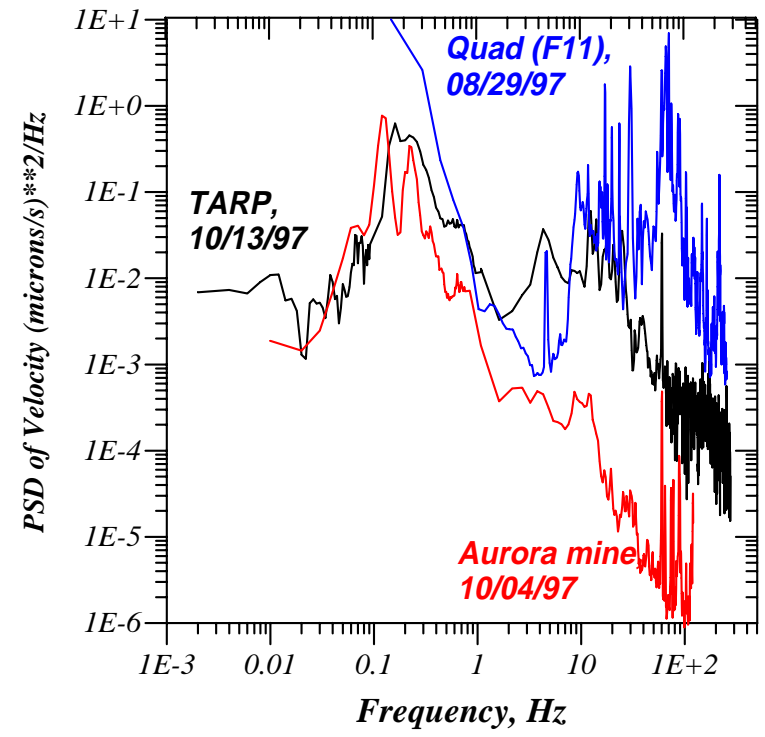

Figure 1: Spectra of ground motion in Aurora mine, TARP tunnel, and the Tevatron magnet vibrations 
Power spectral densities of the ground velocities $S_{v}(f)$ measured in the Aurora mine, in the TARP shaft are presented in Fig. 1 in comparison with the Tevatron quadrupole magnet vibration PSD. These spectra cover five decades of frequency band from $0.005 \mathrm{~Hz}$ to $280 \mathrm{~Hz}$ and are obtained with different probes and with different sampling rates (besides different places and different times). For example, the TARP curve (solid line) consists of spectrum measured by the STS-2 vertical probe (from $0.005 \mathrm{~Hz}$ to $0.1 \mathrm{~Hz}$ ), by the SM3-KV geophone (from $0.1 \mathrm{~Hz}$ to $120 \mathrm{~Hz}$ ) and by the Wiloxon piezo-probe (from 120 to $280 \mathrm{~Hz}$ ). The Aurora data (dashed line) show no vibration spectrum above 120 $\mathrm{Hz}$ - the motion is too small to be detected by the piezoaccelerometers.

One can see that the Aurora mine is the quietest place of the three. Some technologically related peaks are seen in the "Aurora" PSD only at $60-120 \mathrm{~Hz}$ range. We believe that it is due to lighting transformers in the tunnel. Below $0.5 \mathrm{~Hz}$ the spectral density in Aurora mine and in the TARP tunnel are about the same, and are mainly due to microseismic waves. Above $2 \mathrm{~Hz}$, the TARP PSD is $20-800$ times the Aurora mine PSD. Nosier environment on the surface and more technological equipment in the tunnel itself are probable reasons for two very broad peaks in the TARP spectrum at $5 \mathrm{~Hz}$ and around $25 \mathrm{~Hz}$, respectively (as damping decrement of the ground grow with frequency). Finally, the Tevatron quadrupole spectrum consists of many peaks $(4.6 \mathrm{~Hz}, 9.2 \mathrm{~Hz}, 20 \mathrm{~Hz}, 60 \mathrm{~Hz}$, etc.) and is much noisier (as we discussed above - due to the Tevatron equipment) than the others above $10 \mathrm{~Hz}$. All three spectra show "a microseismic peak" near $0.2 \mathrm{~Hz}$. Usually, the rms amplitude of the tunnel motion is less than a micron everywhere, but occasionally we observed ground motion amplitude is of the order of 10-25 microns, like during Oct.14, 1997 M6.8 Chile earthquake. These waves with periods of $20-80 \mathrm{sec}$ were well correlated over maximum distances of our studies (about $80 \mathrm{~m}$ ).

Fig.2 shows real and imaginary parts of the correlation spectrum $C_{x_{1} x_{2}}(f)$ of signals from two vertical SM3-KV geophones placed $75 \mathrm{~m}$ apart in the TARP shaft. Each of the curves is an average over 200 measurements that gives an estimate of the statistical error of about 0.1. The first remarkable feature of the data is that at frequencies below $100 \mathrm{~Hz}$ the real part is much larger than the imaginary one. The latter is almost zero below $10 \mathrm{~Hz}$, while the real part performs some damped oscillations with the frequency increase. Such a behavior is close to prediction of the model in which the vibration sources are uniformly and continuously distributed over ground surface and generate waves at all frequencies without any phase correlation, see e.g. [4]. Under these assumptions the correlation spectrum between signals detected in two points $L$ meters apart is equal to $\operatorname{Re} C(f)=J_{0}(2 \pi L f / v(f)), \quad \operatorname{Im} C(f)=0$, where $v(f)$ is the wave propagation velocity and $J_{0}(x)$ is zeroth order Bessel function. In Fig. 2 we present the fitting curves with parameters $L=75 \mathrm{~m}$ and $v(f)[\mathrm{m} / \mathrm{s}]=3800-4 \cdot f[H z]$.
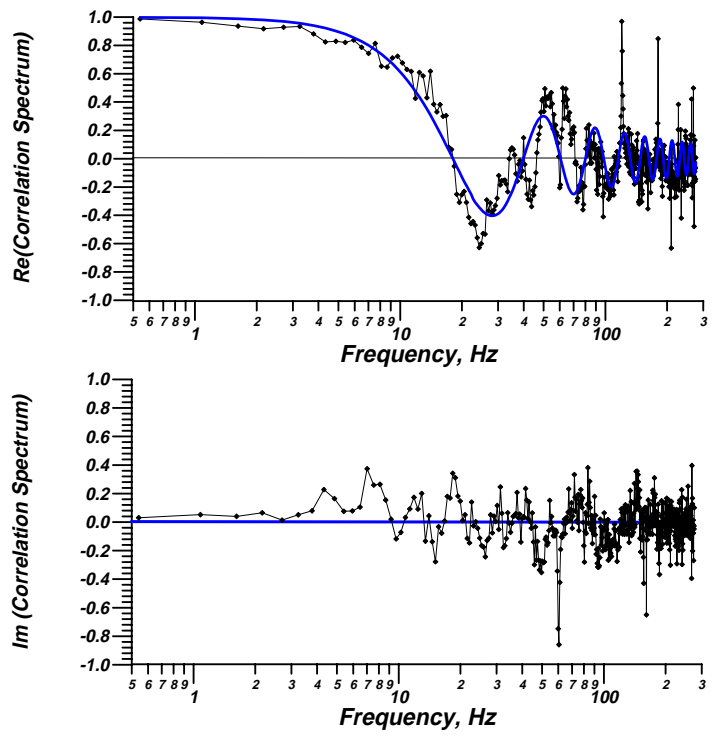

Figure 2: Real and Imaginary parts of correlation spectra measured in the TARP shaft at distance of $75 \mathrm{~m}$ between probes and fit accordingly to random source model.

\section{DISCUSSION AND PLANS}

Integration of the PSDs accordingly to

$$
\sigma_{x}(f)=\int_{f}^{\infty} S_{x}(f) d f=\int_{f}^{\infty} S_{v}(f) \frac{d f}{(2 \pi f)^{2}},
$$

(here $S_{v}(f)$ is the PSD of velocity, $S_{x}(f)=S_{v}(f) / \omega^{2}$ is the PSD of displacement) gives us the rms amplitudes of vibrations. Fig. 3 presents integrated vibration amplitudes in the Aurora mine and on top of the Tevatron quadrupole. One can see that the amplitudes in the deep tunnels are about $0.3 \mu \mathrm{m}$ at frequencies $\sim 0.5 \mathrm{~Hz}$ and below, while above $100 \mathrm{~Hz}$ they are less than $0.1 \mathrm{~nm}=10^{-4} \mu \mathrm{m}$. Motion of the quadrupole is several times larger. Other curves are for the tolerances: for the X-band linear collider it is the ground motion which causes $1.5 \%$ luminosity degradation accordingly to Ref.[4]. We would like to emphasize, that the tolerances for other than than X-band LCs can be much less stringent if larger bunch spacing allows to implement bunch-by-bunch trajectory correction feedback system. The muon collider requirement is presented by the rms amplitude of focusing magnets that leads to beams separation of about $10 \%$ of the rms beam size at the interaction point. The VLHC tolerance consists of two parts [2]: at frequencies below $10 \mathrm{~Hz}$ the curve shows the vibration amplitude that causes the beam orbit vibration amplitude about $10 \%$ of the rms beam size. Above $90 \mathrm{~Hz}$ the line corresponds to requirement on the rms transverse emittance growth less than $0.1 \pi \mathrm{mm} \cdot \mathrm{mrad} / 5$ hours without (solid line) and with feedback system to damp excited betatron oscillations (dashed line, feedback allows to ease the ground motion tolerances some 10 times or more depending on the beam tunespread). One can see that the Aurora mine amplitudes are below all the tolerances, although close to the VLHC ones around 90-120 Hz. In contrast, vibrations of the Tevatron quadrupole are potentially very dangerous for 
all three machines at frequencies below 20-60 Hz (orders of magnitude excess), and several times above the VLHC requirement above $70 \mathrm{~Hz}$.

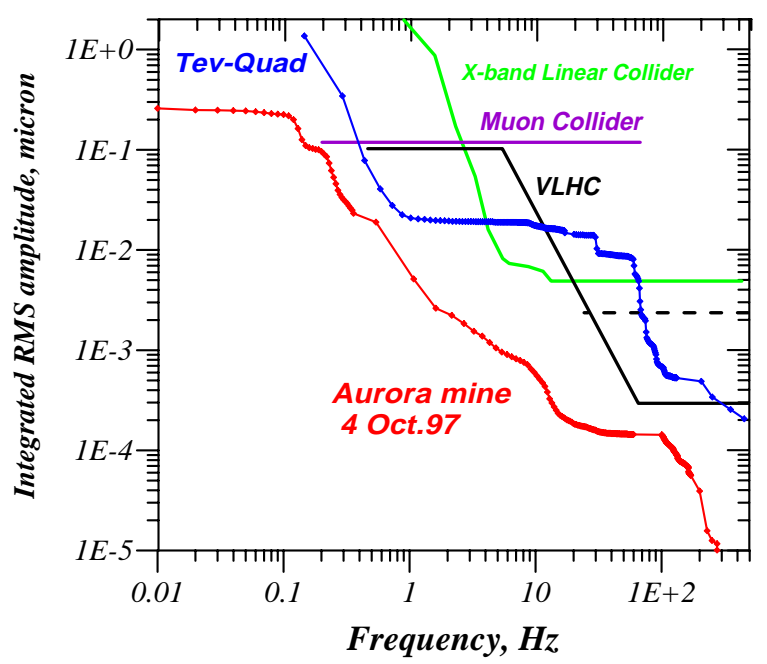

Figure 3: Comparison of the measured ground and quadrupole vibration amplitudes with tolerances for the muon collider, X-band linear collider and the VLHC.

We have to note that, in general, accelerators are relatively 'noisy' sites because of their technical noises. We compare our data with previous measurements at accelerator facilities in Fig.4. It presents the PSDs of ground velocity $S_{v}(f)=S_{x}(f)(2 \pi f)^{2}$ measured in the Aurora mine (marked as FNAL) and in the tunnels of the SLC(SLAC) [4], HERA [5], KEK[6], LEP(CERN)[7], and so-called "New Low Noise Model" [8] - a minimum of geophysical observations worldwide. One can see that the PSDs measured at accelerators are well above the "lownoise" spectrum. At the same time, vibrations in HERA, which is located under populated area in city of Hamburg, are somewhat larger than in the other tunnels.

Main conclusions of our studies are:

1. Ground vibration have been measured at the FNAL site and in deep tunnels outside in wide frequency band from few hundredths of a $\mathrm{Hz}$ to several hundreds $\mathrm{Hz}$. We have observed that vibrations above $1 \mathrm{~Hz}$ are well affected by cultural noise which vary significantly in time and also strongly depend on location and the depth, while below 1 $\mathrm{Hz}$ main contribution to the ground motion comes from natural sources and performs slow temporal variations.

2. Comparison of on-surface and underground sites have shown that levels of vibrations are typically smaller in deep tunnels. Effects due to on-surface noise sources are less seen in the deep tunnels, though visible. Amplitudes of horizontal and vertical vibrations are approximately the same.

3. The maximum amplitudes are observed for the motion of the Tevatron quadrupole magnets when the Tevatron and the Main Ring accelerators were operating. It was somewhat larger than the motion of the tunnel floor nearby. Careful engineering of mechanical supports, of vacuum, power and cooling systems should be an important part of

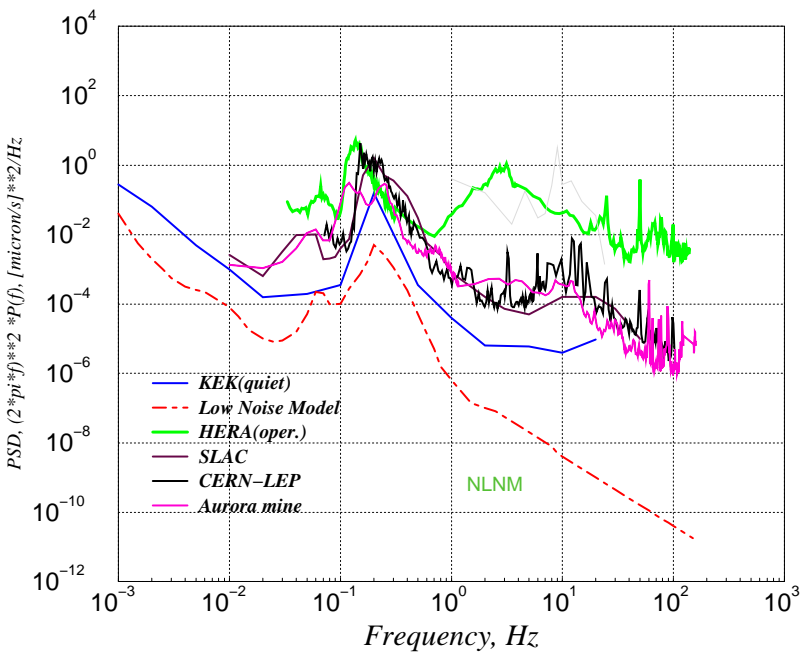

Figure 4: Ground motion spectra at different accelerator sites and the USGS New Low-Noise Model.

R\&D efforts to decrease the level of vibrations in any other future collider.

4. In deep tunnels in the Illinois dolomite (Aurora mine, the TARP shaft) we observed vibrations below the tolerances for all the collider projects, while the Main Ring tunnel is not quiet enough for future colliders, especially for the VLHC.

5. Investigations of a spatial characteristics of the fast ground motion have shown that above $1-4 \mathrm{~Hz}$ the correlation significantly drops at dozens of meters of the distance between points.

Our future plans include measurements of a very slow ground motion (time scale from minutes to a year) in the Tevatron tunnel and in deep tunnels in the Illinois dolomite with use of a hydrostatic leveling system. Experimental data on long term tunnel stability are needed to design orbit correction and alignment systems of future colliders.

Seismic studies presented above would not have been done without help of many people from FNAL, BINP, ANL, SLAC, MWRDGC, Conco Co.. We are indebted to all of them.

\section{REFERENCES}

[1] V.Shiltsev, Proc. EPAC'96, Barcelona(1996), 32.

[2] V. Shiltsev, Preprint FNAL-TM-1987(1996).

[3] B.Baklakov, et. al, Phys. Rev. ST Accel. Beams, 1, 031001 (1998).

[4] Appendix C of NLC ZDR, Report SLAC-R-0485 (1996).

[5] V.Shiltsev, et.al, Proc. PAC'95, Dallas(1995) 2078, 3424.

[6] S.Takeda, M.Yoshioka, KEK-Preprint 95-209 (1996).

[7] V.Jouravlev,et.al, CERN-SL/93-53, CLIC-Note-217 (1993).

[8] J.Peterson, USGS Open-File Report 93-322, Albuquerque, NM (1993). 